\title{
Information Aggregation in Intelligent Systems Using Generalized Operators
}

\author{
Imre J. Rudas, János Fodor
}

\begin{abstract}
Aggregation of information represented by membership functions is a central matter in intelligent systems where fuzzy rule base and reasoning mechanism are applied. Typical examples of such systems consist of, but not limited to, fuzzy control, decision support and expert systems. Since the advent of fuzzy sets a great number of fuzzy connectives, aggregation operators have been introduced. Some families of such operators (like t-norms) have become standard in the field. Nevertheless, it also became clear that these operators do not always follow the real phenomena. Therefore, there is a natural need for finding new operators to develop more sophisticated intelligent systems. This paper summarizes the research results of the authors that have been carried out in recent years on generalization of conventional operators.
\end{abstract}

Keywords: t-norm, t-conorm, uninorm, entropy- and distance-based conjunctions and disjunctions.

\section{Introduction}

Information aggregation is one of the key issues in development of intelligent systems. Although fuzzy set theory provides a host of attractive aggregation operators for integrating the membership values representing uncertain information, the results do not always follow the modeled real phenomena and it has been shown that in some situations some operations may work better than others.

Since the pioneering work of Zadeh the basic research was oriented towards the investigation of the properties of t-norms and t-conorms and also to find new ones satisfying the axiom system. As a result of this a great number (of various type) of t-operators have been introduced accepting the axiom system as a fixed, unchangeable skeleton.

Until the last few years no strong efforts were devoted to generalize t-operators by modifying "weakening" this axiom system. On one hand, the sound theoretical foundation as well as their wide variety have given t-norms and t-conorms almost an exclusive role in different theoretical investigations and practical applications. On the other hand, people are inclined to use them also as a matter of routine. The following observations support this statement and .

When one works with binary conjunctions and there is no need to extend them for three or more arguments, associativity is an unnecessarily restrictive condition. The same is valid for commutativity if the two arguments have different semantical backgrounds and it has no sense to interchange one with the other.

These observations, which are very often left out of consideration, advocate our study and have urged us to revise definitions and properties of operations for information aggregation and reasoning.

\section{Traditional Operations}

The original fuzzy set theory was formulated in terms of Zadeh's standard operations of intersection, union and complement. The axiomatic skeleton used for characterizing fuzzy intersection and fuzzy union are known as triangular norms (t-norms) and triangular conorms ( $t$-conorms), respectively. For more details we refer to the book [9].

\subsection{Triangular Norms and Conorms}

Definition 1. A non-increasing function $N:[0,1] \rightarrow[0,1]$ satisfying $N(0)=1, N(1)=0$ is called a negation. A negation $N$ is called strict if $N$ is strictly decreasing and continuous. A strict negation $N$ is said to be a strong negation if $N$ is also involutive: $N(N(x))=x$ for all $x \in[0,1]$.

The standard negation is simply $N_{s}(x)=1-x, \quad x \in[0,1]$. Clearly, this negation is strong. It plays a key role in the representation of strong negations.

We call a continuous, strictly increasing function $\varphi:[0,1] \rightarrow[0,1]$ with $\varphi(0)=0, \varphi(1)=1$ an automorphism of the unit interval. 
Definition 2. A triangular norm (shortly: a t-norm) is a function $T:[0,1]^{2} \rightarrow[0,1]$ which is associative, increasing and commutative, and satisfies the boundary condition $T(1, x)=x$ for all $x \in[0,1]$.

Definition 3. A triangular conorm (shortly: a t-conorm) is an associative, commutative, increasing $S:[0,1]^{2} \rightarrow$ $[0,1]$ function, with boundary condition $S(0, x)=x$ for all $x \in[0,1]$.

Notice that continuity of a t-norm and a t-conorm is not taken for granted.

In what follows we assume that $T$ is a t-norm, $S$ is a t-conorm and $N$ is a strict negation.

Clearly, for every t-norm $T$ and strong negation $N$, the operation $S$ defined by

$$
S(x, y)=N(T(N(x), N(y))), \quad x, y \in[0,1]
$$

is a t-conorm. In addition, $T(x, y)=N(S(N(x), N(y)))(x, y \in[0,1])$. In this case $S$ and $T$ are called $N$-duals. In case of the standard negation (i.e., when $N(x)=1-x$ for $x \in[0,1]$ ) we simply speak about duals. Obviously, equality (1) expresses the De Morgan's law in the fuzzy case.

Generally, for any t-norm $T$ and t-conorm $S$ we have

$$
T_{W}(x, y) \leq T(x, y) \leq T_{M}(x, y) \quad \text { and } \quad S_{M}(x, y) \leq S(x, y) \leq S_{S}(x, y),
$$

where $T_{M}(x, y)=\min (x, y), S_{M}(x, y)=\max (x, y), T_{W}$ is the weakest t-norm, and $S_{S}$ is the strongest t-conorm.

These inequalities are important from practical point of view as they establish the boundaries of the possible range of mappings $T$ and $S$.

\subsection{Uninorms and Nullnorms}

\section{Uninorms}

Uninorms were introduced by Yager and Rybalov [19] as a generalization of t-norms and t-conorms. For uninorms, the neutral element is not forced to be either 0 or 1 , but can be any value in the unit interval.

Definition 4. [19] A uninorm $U$ is a commutative, associative and increasing binary operator with a neutral element $e \in[0,1]$, i.e., for all $x \in[0,1]$ we have $U(x, e)=x$.

T-norms do not allow low values to be compensated by high values, while t-conorms do not allow high values to be compensated by low values. Uninorms may allow values separated by their neutral element to be aggregated in a compensating way. The structure of uninorms was studied by Fodor et al. [11]. For a uninorm $U$ with neutral element $e \in] 0,1]$, the binary operator $T_{U}$ defined by

$$
T_{U}(x, y)=\frac{U(e x, e y)}{e}
$$

is a t-norm; for a uninorm $U$ with neutral element $e \in\left[0,1\left[\right.\right.$, the binary operator $S_{U}$ defined by

$$
S_{U}(x, y)=\frac{U(e+(1-e) x, e+(1-e) y)-e}{1-e}
$$

is a t-conorm. The structure of a uninorm with neutral element $e \in] 0,1\left[\right.$ on the squares $[0, e]^{2}$ and $[e, 1]^{2}$ is therefore closely related to t-norms and t-conorms. For $e \in] 0,1\left[\right.$, we denote by $\phi_{e}$ and $\psi_{e}$ the linear transformations defined by $\phi_{e}(x)=\frac{x}{e}$ and $\psi_{e}(x)=\frac{x-e}{1-e}$. To any uninorm $U$ with neutral element $\left.e \in\right] 0,1[$, there corresponds a t-norm $T$ and a t-conorm $S$ such that:

(i) for any $(x, y) \in[0, e]^{2}: U(x, y)=\phi_{e}^{-1}\left(T\left(\phi_{e}(x), \phi_{e}(y)\right)\right)$;

(ii) for any $(x, y) \in[e, 1]^{2}: U(x, y)=\psi_{e}^{-1}\left(S\left(\psi_{e}(x), \psi_{e}(y)\right)\right)$.

On the remaining part of the unit square, i.e. on $E=[0, e[\times] e, 1] \cup] e, 1] \times[0, e[$, it satisfies

$$
\min (x, y) \leq U(x, y) \leq \max (x, y),
$$

and could therefore partially show a compensating behaviour, i.e. take values strictly between minimum and maximum. Note that any uninorm $U$ is either conjunctive, i.e. $U(0,1)=U(1,0)=0$, or disjunctive, i.e. $U(0,1)=$ $U(1,0)=1$. 


\section{Representation of Uninorms}

In analogy to the representation of continuous Archimedean t-norms and t-conorms in terms of additive generators, Fodor et al. [11] have investigated the existence of uninorms with a similar representation in terms of a single-variable function. This search leads back to Dombi's class of aggregative operators [7]. This work is also closely related to that of Klement $e t$ al. on associative compensatory operators [15]. Consider $e \in] 0,1[$ and a strictly increasing continuous $[0,1] \rightarrow \overline{\mathbb{R}}$ mapping $h$ with $h(0)=-\infty, h(e)=0$ and $h(1)=+\infty$. The binary operator $U$ defined by

$$
U(x, y)=h^{-1}(h(x)+h(y))
$$

for any $(x, y) \in[0,1]^{2} \backslash\{(0,1),(1,0)\}$, and either $U(0,1)=U(1,0)=0$ or $U(0,1)=U(1,0)=1$, is a uninorm with neutral element $e$. The class of uninorms that can be constructed in this way has been characterized [11].

Consider a uninorm $U$ with neutral element $e \in] 0,1[$, then there exists a strictly increasing continuous $[0,1] \rightarrow$ $\overline{\mathbb{R}}$ mapping $h$ with $h(0)=-\infty, h(e)=0$ and $h(1)=+\infty$ such that

$$
U(x, y)=h^{-1}(h(x)+h(y))
$$

for any $(x, y) \in[0,1]^{2} \backslash\{(0,1),(1,0)\}$ if and only if

(i) $U$ is strictly increasing and continuous on $] 0,1[2$;

(ii) there exists an involutive negator $N$ with fixpoint $e$ such that

$$
U(x, y)=N(U(N(x), N(y))))
$$

for any $(x, y) \in[0,1]^{2} \backslash\{(0,1),(1,0)\}$.

The uninorms characterized above are called representable uninorms. The mapping $h$ is called an additive generator of $U$. The involutive negator corresponding to a representable uninorm $U$ with additive generator $h$, as mentioned in condition (ii) above, is denoted $N_{U}$ and is given by

$$
N_{U}(x)=h^{-1}(-h(x))
$$

Clearly, any representable uninorm comes in a conjunctive and a disjunctive version, i.e. there always exist two representable uninorms that only differ in the points $(0,1)$ and $(1,0)$. Representable uninorms are almost continuous, i.e. continuous except in $(0,1)$ and $(1,0)$, and Archimedean, in the sense that $(\forall x \in] 0, e[)(U(x, x)<x)$ and $(\forall x \in] e, 1[)(U(x, x)>x)$. Clearly, representable uninorms are not idempotent. The classes $U_{\min }$ and $U_{\max }$ do not contain representable uninorms. A very important fact is that the underlying t-norm and t-conorm of a representable uninorm must be strict and cannot be nilpotent. Moreover, given a strict t-norm $T$ with decreasing additive generator $f$ and a strict t-conorm $S$ with increasing additive generator $g$, we can always construct a representable uninorm $U$ with desired neutral element $e \in] 0,1[$ that has $T$ and $S$ as underlying t-norm and t-conorm. It suffices to consider as additive generator the mapping $h$ defined by

$$
h(x)=\left\{\begin{array}{cl}
-f\left(\frac{x}{e}\right) & , \text { if } x \leq e \\
g\left(\frac{x-e}{1-e}\right) & , \text { if } x \geq e
\end{array} .\right.
$$

On the other hand, the following property indicates that representable uninorms are in some sense also generalizations of nilpotent t-norms and nilpotent t-conorms: $(\forall x \in[0,1])\left(U\left(x, N_{U}(x)\right)=N_{U}(e)\right)$. This claim is further supported by studying the residual operators of representable uninorms in [6].

As an example of the representable case, consider the additive generator $h$ defined by $h(x)=\log \frac{x}{1-x}$, then the corresponding conjunctive representable uninorm $\mathcal{U}$ is given by $U(x, y)=0$ if $(x, y) \in\{(1,0),(0,1)\}$, and

$$
U(x, y)=\frac{x y}{(1-x)(1-y)+x y}
$$

otherwise, and has as neutral element $\frac{1}{2}$. Note that $N_{U}$ is the standard negator: $N_{U}(x)=1-x$.

The class of representable uninorms contains famous operators, such as the functions for combining certainty factors in the expert systems MYCIN (see [18, 5]) and PROSPECTOR [5]. The MYCIN expert system was one 
of the first systems capable of reasoning under uncertainty [2]. To that end, certainty factors were introduced as numbers in the interval $[-1,1]$. Essential in the processing of these certainty factors is the modified combining function $C$ proposed by van Melle [2]. The $[-1,1]^{2} \rightarrow[-1,1]$ mapping $C$ is defined by

$$
C(x, y)=\left\{\begin{array}{cl}
x+y(1-x) & , \text { if } \min (x, y) \geq 0 \\
x+y(1+x) & , \text { if } \max (x, y) \leq 0 \\
\frac{x+y}{1-\min (|x|,|y|)} & , \text { otherwise }
\end{array}\right.
$$

The definition of $C$ is not clear in the points $(-1,1)$ and $(1,-1)$, though it is understood that $C(-1,1)=C(1,-1)=$ -1 . Rescaling the function $C$ to a binary operator on $[0,1]$, we obtain a representable uninorm with neutral element $\frac{1}{2}$ and as underlying t-norm and t-conorm the product and the probabilistic sum. Implicitly, these results are contained in the book of Hájek et al. [14], in the context of ordered Abelian groups.

\section{Nullnorms}

Definition 5. [3] A nullnorm $V$ is a commutative, associative and increasing binary operator with an absorbing element $a \in[0,1]$, i.e. $(\forall x \in[0,1])(V(x, a)=a)$, and that satisfies

$$
\begin{aligned}
& (\forall x \in[0, a])(V(x, 0)=x) \\
& (\forall x \in[a, 1])(V(x, 1)=x)
\end{aligned}
$$

The absorbing element $a$ corresponding to a nullnorm $V$ is clearly unique. By definition, the case $a=0$ leads back to t-norms, while the case $a=1$ leads back to t-conorms. In the following proposition, we show that the structure of a nullnorm is similar to that of a uninorm. In particular, it can be shown that it is built up from a t-norm, a t-conorm and the absorbing element [3].

Theorem 6. Consider $a \in[0,1]$. A binary operator $V$ is a nullnorm with absorbing element a if and only if

(i) if $a=0: V$ is a t-norm;

(ii) if $0<a<1$ : there exists a $t$-norm $T_{V}$ and a $t$-conorm $S_{V}$ such that $V(x, y)$ is given by

$$
\begin{cases}\phi_{a}^{-1}\left(S_{V}\left(\phi_{a}(x), \phi_{a}(y)\right)\right) & , \text { if }(x, y) \in[0, a]^{2} \\ \psi_{a}^{-1}\left(T_{V}\left(\psi_{a}(x), \psi_{a}(y)\right)\right) & , \text { if }(x, y) \in[a, 1]^{2} \\ a & , \text { elsewhere }\end{cases}
$$

(iii) if $a=1: V$ is a $t$-conorm.

Recall that for any t-norm $T$ and t-conorm $S$ it holds that $T(x, y) \leq \min (x, y) \leq \max (x, y) \leq S(x, y)$, for any $(x, y) \in[0,1]^{2}$. Hence, for a nullnorm $V$ with absorbing element $a$ it holds that $\left(\forall(x, y) \in[0, a]^{2}\right)(V(x, y) \geq$ $\max (x, y))$ and $\left(\forall(x, y) \in[a, 1]^{2}\right)(V(x, y) \leq \min (x, y))$. Clearly, for any nullnorm $V$ with absorbing element $a$ it holds for all $x \in[0,1]$ that

$$
V(x, 0)=\min (x, a) \quad \text { and } \quad V(x, 1)=\max (x, a) .
$$

Notice that, without the additional conditions (4) and (5), it cannot be shown that a commutative, associative and increasing binary operator $V$ with absorbing element $a$ behaves as a t-conorm and t-norm on the squares $[0, a]^{2}$ and $[a, 1]^{2}$.

Nullnorms are a generalization of the well-known median studied by Fung and Fu [13], which corresponds to the case $T=\min$ and $S=\max$. For a more general treatment of this operator, we refer to [10]. We recall here the characterization of that median as given by Czogala and Drewniak [4]. Firstly, they observe that an idempotent, associative and increasing binary operator $O$ has as absorbing element $a \in[0,1]$ if and only if $O(0,1)=O(1,0)=a$. Then the following theorem can be proven.

Theorem 7. [4] Consider $a \in[0,1]$. A continuous, idempotent, associative and increasing binary operator $O$ satisfies $O(0,1)=O(1,0)=a$ if and only if it is given by

$$
O(x, y)=\left\{\begin{array}{cl}
\max (x, y) & , \text { if }(x, y) \in[0, a]^{2} \\
\min (x, y) & , \text { if }(x, y) \in[a, 1]^{2} \\
a & , \text { elsewhere }
\end{array}\right.
$$


Nullnorms are also a special case of the class of $T-S$ aggregation functions introduced and studied by Fodor and Calvo [12].

Definition 8. Consider a continuous t-norm $T$ and a continuous t-conorm $S$. A binary operator $F$ is called a $T-S$ aggregation function if it is increasing and commutative, and satisfies the boundary conditions

$$
\begin{gathered}
(\forall x \in[0,1])(F(x, 0)=T(F(1,0), x)) \\
(\forall x \in[0,1])(F(x, 1)=S(F(1,0), x)) .
\end{gathered}
$$

When $T$ is the algebraic product and $S$ is the probabilistic sum, we recover the class of aggregation functions studied by Mayor and Trillas [17]. Rephrasing a result of Fodor and Calvo, we can state that the class of associative $T-S$ aggregation functions coincides with the class of nullnorms with underlying t-norm $T$ and t-conorm $S$.

\subsection{The Role of Commutativity and Associativity}

One possible way of simplification of axiom skeletons of t-norms and t-conorms may be not requiring that these operations to have the commutative and the associative properties. Non-commutative and non-associative operations are widely used in mathematics, so, why do we restrict our investigations by keeping these axioms? What are the requirements of the most typical applications?

From theoretical point of view the commutative law is not required, while the associative law is necessary to extend the operation to more than two variables. In applications, like fuzzy logic control, fuzzy expert systems and fuzzy systems modeling fuzzy rule base and fuzzy inference mechanism are used, where the information aggregation is performed by operations. The inference procedures do not always require commutative and associative laws of the operations used in these procedures. These properties are not necessary for conjunction operations used in the simplest fuzzy controllers with two inputs and one output. For rules with greater amount of inputs and outputs these properties are also not required if the sequence of variables in the rules are fixed.

Moreover, the non-commutativity of conjunction may in fact be desirable for rules because it can reflect different influences of the input variables on the output of the system. For example, in fuzzy control, the positions of the input variables the "error" and the "change in error" in rules are usually fixed and these variables have different influences on the output of the system. In the application areas of fuzzy models when the sequence of operands is not fixed, the property of non-commutativity may not be desirable. Later some examples will be given for parametric non-commutative and non-associative operations.

\section{Generalized Conjunctions and Disjunctions}

The axiom systems of t-norms and t-conorms are very similar to each other except the neutral element, i.e. the type is characterized by the neutral element. If the neutral element is equal to 1 then the operation is a conjunction type, while if the neutral element is zero the disjunction operation is obtained. By using these properties we introduce the concepts of conjunction and disjunction operations [1].

Definition 9. Let $T$ be a mapping $T:[0,1] \times[0,1] \rightarrow[0,1] . T$ is a conjunction operation if $T(x, 1)=x$ for all $x \in[0,1]$.

Definition 10. Let $S$ be a mapping $S:[0,1] \times[0,1] \rightarrow[0,1] . S$ is a conjunction operation if $S(x, 0)=x$ for all $x \in[0,1]$.

Conjunction and disjunction operations may also be obtained one from another by means of an involutive negation $N: S(x, y)=N(T(N(x), N(y)))$, and $T(x, y)=N(S(N(x), N(y)))$.

It can be seen easily that conjunction and disjunction operations satisfy the following boundary conditions: $T(1,1)=1, T(0, x)=T(x, 0)=0, S(0,0)=0, S(1, x)=S(x, 1)=1$. By fixing these conditions, new types of generalized operations are introduced.

Definition 11. Let $T$ be a mapping $T:[0,1] \times[0,1] \rightarrow[0,1] . T$ is a quasi-conjunction operation if $T(0,0)=$ $T(0,1)=T(1,0)=0$, and $T(1,1)=1$.

Definition 12. Let $S$ be a mapping $S:[0,1] \times[0,1] \rightarrow[0,1] . S$ is a quasi-disjunction operation if $S(0,1)=S(1,0)=$ $S(1,1)=1$, and $S(0,0)=0$. 
It is easy to see that conjunction and disjunction operations are quasi-conjunctions and quasi-disjunctions, respectively, but the converse is not true.

Omitting $T(1,1)=1$ and $S(0,0)=0$ from the definitions further generalization can be obtained.

Definition 13. Let $T$ be a mapping $T:[0,1] \times[0,1] \rightarrow[0,1]$. $T$ is a pseudo-conjunction operation if $T(0,0)=$ $T(0,1)=T(1,0)=0$.

Definition 14. Let $S$ be a mapping $S:[0,1] \times[0,1] \rightarrow[0,1] . S$ is a pseudo-disjunction operation if $S(0,1)=$ $S(1,0)=S(1,1)=1$.

Theorem 15. Assume that $T$ and $S$ are non-decreasing pseudo-conjunctions and pseudo-disjunctions, respectively. Then there exist the absorbing elements 0 and 1 such as $T(x, 0)=T(0, x)=0$ and $S(x, 1)=S(1, x)=1$.

\subsection{Entropy-based Conjunction and Disjunction Operators}

The question of how fuzzy is a fuzzy set has been one of the issues associated with the development of the fuzzy set theory. In accordance with a current terminological trend in the literature, measure of uncertainty is being referred as measure of fuzziness, or fuzzy entropy [16].

Throughout this part the following notations will be used; $X$ is the universal set, $\boldsymbol{F}(X)$ is the class of all fuzzy subsets of $X, \Re^{+}$is the set of non negative real numbers, $\bar{A}$ is the fuzzy complement of $A \in \boldsymbol{F}(X)$ and $|A|$ is the cardinality of $A$.

Definition 16. Let $X$ be a universal set and $A$ is a fuzzy subset of $X$ with membership function $\mu_{A}$. The fuzzy entropy is a mapping $e: \boldsymbol{F}(X) \rightarrow \mathfrak{R}^{+}$which satisfies the following axioms:

AE $1 e(A)=0$ if $A$ is a crisp set.

AE 2 If $A \prec B$ then $e(A) \leq e(B)$; where $A \prec B$ means that $A$ is sharper than $B$.

AE $3 e(A)$ assumes its maximum value if and only if $A$ is maximally fuzzy.

AE $4 e(A)=e(\bar{A}), \forall A \in X$.

Let $e_{p}$ be equilibrium of the fuzzy complement $C$ and specify $\mathbf{A E} 2$ and AE 3 as follows:

AES $2 A$ is sharper than $B$ in the following sense:

$\mu_{A}(x) \leq \mu_{B}(x)$ for $\mu_{B}(x) \leq e_{p}$ and $\mu_{A}(x) \geq \mu_{B}(x)$ for $\mu_{B}(x) \geq e_{p}$, for all $x \in X$.

AES $3 A$ is defined maximally fuzzy when $\mu_{A}(x)=e_{p} \forall x \in X$.

Let $A$ be a fuzzy subset of $X$ and define the following function $f_{A}: X \rightarrow[0,1]$ by

$$
f_{A}: x \mapsto \begin{cases}\mu_{A}(x) & \text { if } \mu_{A}(x) \leq e_{p} \\ C\left(\mu_{A}(x)\right) & \text { if } \mu_{A}(x)>e_{p}\end{cases}
$$

Denote $\Phi_{A}$ the fuzzy set generated by $f_{A}$ as its membership function.

Theorem 17. The $g\left(\left|\Phi_{A}\right|\right)$ is an entropy, where $g: \Re \rightarrow \Re$ is a monotonically increasing real function and $g(0)=0$.

Definition 18. Let $A$ be a fuzzy subset of $X . f_{A}$ is said to be an elementary fuzzy entropy function if the cardinality of the fuzzy set $\Phi_{A}=\left\{\left(x, f_{A}(x)\right) \mid x \in X, f_{A}(x) \in[0,1]\right\}$ is an entropy of $A$.

It is obvious that $f_{A}$ is an elementary entropy function.

Now we introduce some operations based on entropy. For more details we refer to [1].

Definition 19. Let $A$ and $B$ be two fuzzy subsets of the universe of discourse $X$ and denote $\varphi_{A}$ and $\varphi_{B}$ their elementary entropy functions, respectively. The minimum entropy conjunction operations is defined as $I_{\varphi}^{*}=I_{\varphi}^{*}(A, B)=$ $\left\{\left(x, \mu_{I_{\varphi}^{*}}(x)\right) \mid x \in X, \mu_{I_{\varphi}^{*}}(x) \in[0,1]\right\}$, where

$$
\mu_{I_{\varphi}^{*}}: x \mapsto\left\{\begin{array}{cl}
\mu_{A}(x), & \text { if } \varphi_{A}(x)<\varphi_{B}(x) \\
\mu_{B}(x), & \text { if } \varphi_{B}(x)<\varphi_{A}(x) \\
\min \left(\mu_{A}(x), \mu_{B}(x)\right), & \text { if } \varphi_{A}(x)=\varphi_{B}(x)
\end{array} .\right.
$$

Definition 20. Let $A$ and $B$ be two fuzzy subsets of the universe of discourse $X$ and denote $\varphi_{A}$ and $\varphi_{B}$ their elementary entropy functions, respectively. The maximum entropy disjunction operation is defined as $U_{\varphi}^{*}=U_{\varphi}^{*}(A, B)=$ $\left\{\left(x, \mu_{U_{\varphi}^{*}}(x)\right) \mid x \in X, \mu_{U_{\varphi}^{*}}(x) \in[0,1]\right\}$, where 

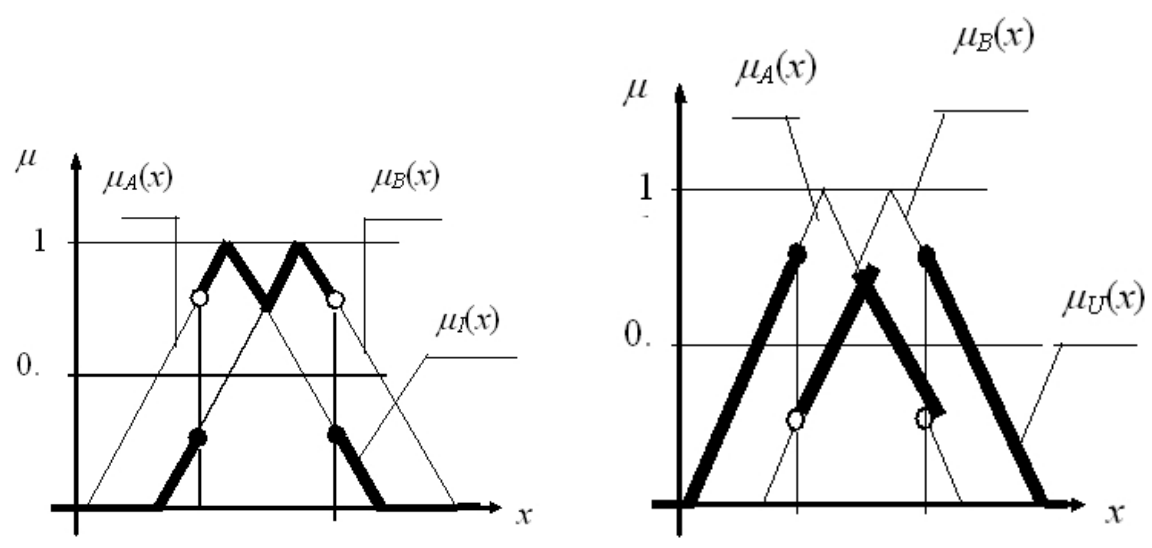

Figure 1: Entropy based conjunction operator (left) and entropy based disjunction operator (right)
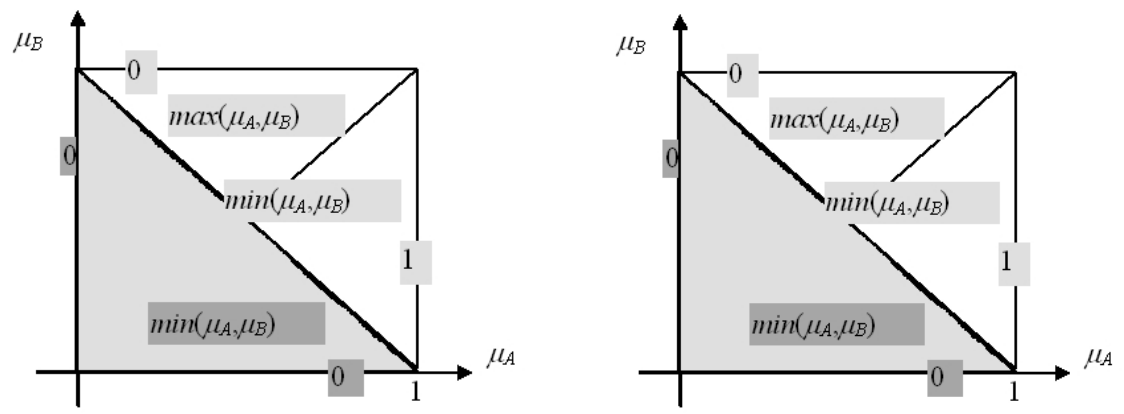

Figure 2: The construction of $I_{\varphi}^{*}$ (left) and the construction of $U_{\varphi}^{*}$ (right).

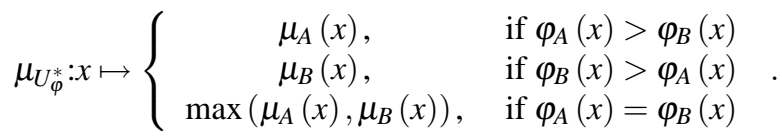

The geometrical representation of the minimum fuzziness conjunction and the maximum fuzziness disjunction operators can be seen in Fig. 1.

Several important properties of these operations as well as their construction can be found in [1]. Now we present only two figures about the construction.

Notice also that $I_{\varphi}^{*}$ is a quasi-conjunction, $U_{\varphi}^{*}$ is a quasi-disjunction operation, and $U_{\varphi}^{*}$ is a commutative semigroup operation on $[0,1][1]$.

\subsection{A Parametric Family of Quasi-Conjunctions}

Let us cite the following result, which is the base of the forthcoming parametric construction, from [1].

Theorem 21. Suppose $T_{1}, T_{2}$ are quasi-conjunctions, $S_{1}$ and $S_{2}$ are pseudo disjunctions and $h, g_{1}, g_{2}:[0,1] \rightarrow$ $[0,1]$ are non-decreasing functions such that $g_{1}(1)=g_{2}(1)=1$. Then the following functions

$$
\begin{gathered}
T(x, y)=T_{2}\left(T_{1}(x, y), S_{1}\left(g_{1}(x), g_{2}(y)\right)\right) \\
T(x, y)=T_{2}\left(T_{1}(x, y), g_{1} S_{1}(x, y)\right) \\
T(x, y)=T_{2}\left(T_{1}(x, y), S_{2}\left(h(x), S_{1}(x, y)\right)\right)
\end{gathered}
$$

are quasi-conjunctions. 
By the use of this Theorem the simplest parametric quasi-conjunction operations can be obtained as follows:

$$
\begin{gathered}
T(x, y)=x^{p} y^{q}, \\
T(x, y)=\min \left(x^{p}, y^{q}\right), \\
T(x, y)=(x y)^{p}(x+y-x y)^{q}
\end{gathered}
$$

where $p, q \geq 0$.

\section{Distance-based Operations}

Let $e$ be an arbitrary element of the closed unit interval $[0,1]$ and denote by $d(x, y)$ the distance of two elements $x$ and $y$ of $[0,1]$. The idea of definitions of distance-based operators is generated from the reformulation of the definition of the min and max operators as follows

$$
\min (x, y)=\left\{\begin{array}{ll}
x, & \text { if } d(x, 0) \leq d(y, 0) \\
y, & \text { if } d(x, 0)>d(y, 0)
\end{array}, \quad \max (x, y)= \begin{cases}x, & \text { if } d(x, 0) \geq d(y, 0) \\
y, & \text { if } d(x, 0)<d(y, 0)\end{cases}\right.
$$

Based on this observation the following definitions can be introduced, see [1].

Definition 22. The maximum distance minimum operator with respect to $e \in[0,1]$ is defined as

$$
\min _{e}(x, y)=\left\{\begin{array}{cl}
x, & \text { if } d(x, e)>d(y, e) \\
y, & \text { if } d(x, e)<d(y, e) \\
\min (x, y), & \text { if } d(x, e)=d(y, e)
\end{array} .\right.
$$

Definition 23. The maximum distance maximum operator with respect to $e \in[0,1]$ is defined as

$$
\max _{e}(x, y)=\left\{\begin{array}{cl}
x, & \text { if } d(x, e)>d(y, e) \\
y, & \text { if } d(x, e)<d(y, e) \\
\max (x, y), & \text { if } d(x, e)=d(y, e)
\end{array} .\right.
$$

Definition 24. The minimum distance minimum operator with respect to $e \in[0,1]$ is defined as

$$
\min _{e}(x, y)=\left\{\begin{array}{cl}
x, & \text { if } d(x, e)<d(y, e) \\
y, & \text { if } d(x, e)>d(y, e) \\
\min (x, y), & \text { if } d(x, e)=d(y, e)
\end{array} .\right.
$$

Definition 25. The minimum distance maximum operator with respect to $e \in[0,1]$ is defined as

$$
\operatorname{mix}_{e}(x, y)=\left\{\begin{array}{cl}
x, & \text { if } d(x, e)<d(y, e) \\
y, & \text { if } d(x, e)>d(y, e) \\
\max (x, y), & \text { if } d(x, e)=d(y, e)
\end{array} .\right.
$$

\subsection{The Structure of Distance-based Operators}

It can be proved by simple computation that if the distance of $x$ and yis defined as $d(x, y)=|x-y|$ then the distance-based operators can be expressed by means of the min and max operators as follows.

$$
\begin{aligned}
& \min _{e}=\left\{\begin{array}{ll}
\max (x, y), & \text { if } y>2 e-x \\
\min (x, y), & \text { if } y<2 e-x \\
\min (x, y), & \text { if } y=2 e-x
\end{array},\right. \\
& \max _{e}=\left\{\begin{array}{ll}
\min (x, y), & \text { if } y>2 e-x \\
\min (x, y), & \text { if } y<2 e-x \\
\max (x, y), & \text { if } y=2 e-x
\end{array}, \quad \min _{e}= \begin{cases}\min (x, y), & \text { if } y>2 e-x \\
\max (x, y), & \text { if } y<2 e-x \\
\min (x, y), & \text { if } y=2 e-x\end{cases} \right.
\end{aligned}
$$

The structures of the $\max _{e}^{\min }$ and the $\min _{e}^{\min }$ operators are illustrated in Fig. 3 . 

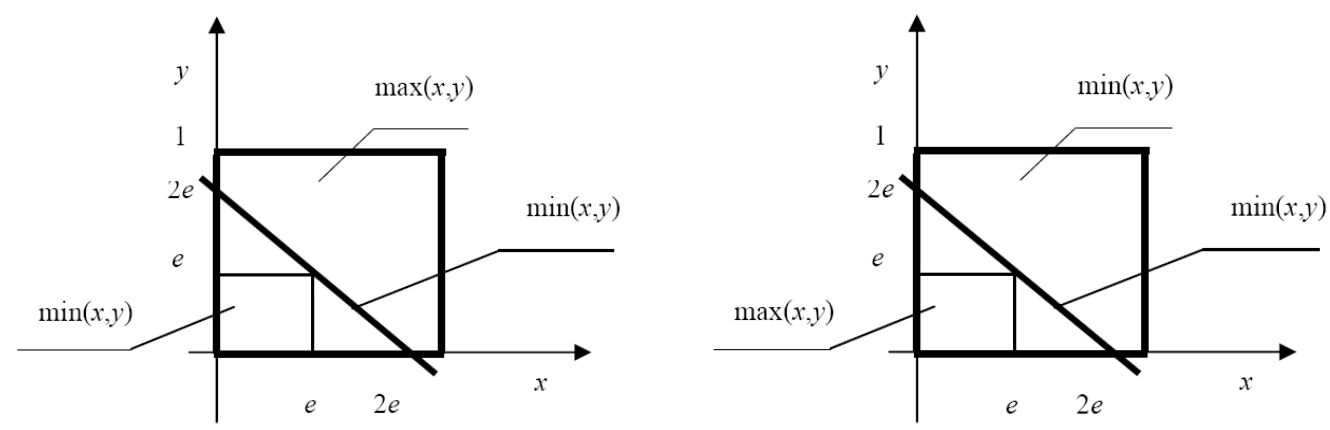

Figure 3: Maximum distance minimum operator (left) and minimum distance minimum operator (right).

\section{Summary and Conclusions}

In this paper we summarized some of our contributions to the theory of non-conventional aggregation operators. Further details and another classes of aggregation operators can be found in [1].

\section{References}

[1] I. Batyrshin, O. Kaynak, and I. Rudas, Fuzzy Modeling Based on Generalized Conjunction Operations, IEEE Transactions on Fuzzy Systems, Vol. 10, No. 5 (2002), pp. 678-683.

[2] B. Buchanan and E. Shortliffe, Rule-Based Expert Systems, The MYCIN Experiments of the Stanford Heuristic Programming Project, Addison-Wesley, Reading, MA, 1984.

[3] T. Calvo, B. De Baets and J. Fodor, The functional equations of Frank and Alsina for uninorms and nullnorms, Fuzzy Sets and Systems 120 (2001) 385-394.

[4] E. Czogala and J. Drewniak, Associative monotonic operations in fuzzy set theory, Fuzzy Sets and Systems 12 (1984), 249-269.

[5] B. De Baets and J. Fodor, Van Melle's combining function in MYCIN is a representable uninorm: An alternative proof. Fuzzy Sets and Systems 104 (1999) 133-136.

[6] B. De Baets and J. Fodor, Residual operators of uninorms, Soft Computing 3 (1999), 89-100.

[7] J. Dombi, Basic concepts for the theory of evaluation: the aggregative operator, European J. Oper. Res. 10 (1982), 282-293.

[8] J.C. Fodor, A new look at fuzzy connectives, Fuzzy Sets and Systems 57 (1993) 141-148.

[9] J.C. Fodor and M. Roubens, Fuzzy Preference Modelling and Multicriteria Decision Support, (Kluwer, Dordrecht, 1994).

[10] J. Fodor, An extension of Fung-Fu's theorem, Internat. J. Uncertain. Fuzziness Knowledge-Based Systems 4 (1996), 235-243.

[11] J. Fodor, R. Yager and A. Rybalov, Structure of uninorms, Internat. J. Uncertain. Fuzziness Knowledge-Based Systems 5 (1997) 411-427.

[12] J. Fodor and T. Calvo, Aggregation functions defined by t-norms and t-conorms, Aggregation and Fusion of Imperfect Information (B. Bouchon-Meunier, ed.), Physica-Verlag, 1998, pp. 36-48.

[13] L. Fung and K. Fu, An axiomatic approach to rational decision-making in a fuzzy environment, Fuzzy Sets and their Applications to Cognitive and Decision Processes (K. Tanaka, L. Zadeh, K. Fu and M. Shimura, eds.), Academic Press, New York, San Francisco, London, 1975, pp. 227-256. 
[14] P. Hájek, T. Havránek and R. Jiroušek, Uncertain Information Processing in Expert Systems (CRC Press, 1992).

[15] E.-P. Klement, R. Mesiar and E. Pap, On the relationship of associative compensatory operators to triangular norms and conorms, Internat. J. Uncertain. Fuzziness Knowledge-Based Systems 4 (1996), 129-144.

[16] J.G. Klir and T.A. Folger, Fuzzy Sets, Uncertainty, and Information (Prentice-Hall International Editions, USA, 1988).

[17] G. Mayor and E. Trillas, On the representation of some aggregation functions, Proc. Internat. Symposium on Multiple-Valued Logic, 1986, pp. 110-114.

[18] A. Tsadiras and K. Margaritis, The MYCIN certainty factor handling function as uninorm operator and its use as a threshold function in artificial neurons, Fuzzy Sets and Systems 93 (1998), 263-274.

[19] R. Yager and A. Rybalov, Uninorm aggregation operators, Fuzzy Sets and Systems 80 (1996) 111-120.

Imre J. Rudas, János Fodor Budapest Tech

Institute of Intelligent Engineering Systems Address: Bécsi út 96/b, H-1034 Budapest, Hungary E-mail: \{rudas,fodor\}@bmf.hu

The research has been supported in part by OTKA T046762.

\section{Editor's note about the authors:}

Imre J. Rudas graduated from Bánki Donát Polytechnic, Budapest in 1971, received the Master Degree in Mathematics from the Eötvös Loránd University, Budapest, the Ph.D. in Robotics from the Hungarian Academy of Sciences in 1987, while the Doctor of Science degree from the Hungarian Academy of Sciences. He received his first Doctor Honoris Causa degree from the Technical University of Kosice, Slovakia and his second Honorary Doctorate from University Polytechnica Timisoara, Romania. He is active as a full university professor and Head of Department of Intelligent Engineering Systems. He serves as the Rector of Budapest Tech from August 1, 2003 for a period of four years. He is a Fellow of IEEE, Administrative Committee member of IEEE Industrial Electronics Society, Chairman of the Hungarian Chapters of IEEE Computational Intelligence and IEEE Systems, Man and Cybernetics Societies. He is also a registered expert of the United Nations Industrial Development Organization and the EU. He is the President of the Hungarian Fuzzy Association and Steering Committee Member of the Hungarian Robotics Association and the John von Neumann Computer Society. He is member of the editorial boards of several leading international scientific journals and founder of the IEEE International Conference Series on Intelligent Engineering Systems and IEEE International Conference on Computational Cybernetics, and some regional symposia. His present areas of research activity are: Robotics with special emphasis on Robot Control, Soft Computing, Computed Aided Process Planning, Fuzzy Control and Fuzzy Sets. He has published one book, more than 300 papers in various journals and international conference proceedings. 
János Fodor obtained his master degree in Mathematics in 1981, his university doctorate in 1984. In 2000 he habilitated at the Roland Eötvös University, Budapest, Hungary. He defended his CSc and DSc degrees in 1991 and 2004, respectively, at the Hungarian Academy of Sciences. In 1997 he won the "Széchenyi Professorship" in the first phase of this award. At the beginning of his researcher's activity he was involved in the stochastic problems of operations research, later on in the field of fuzzy logics, preference modeling, decision making, computational intelligence, intelligent control, and mathematical foundations of the management of uncertainties. He is author of two monographs (issued by Kluwer and Springer), and about 200 other scientific publications. He served as the editor of two scientific books, and serves as the editor of various journal issues. His works obtained more than 700 independent citations. He is member of the editorial boards of various leading international scientific journals. János Fodor is the Co-Chairman of the Hungarian Fuzzy Association, and the EUROFUSE EURO Working Group on Fuzzy Sets. He is member of the Operation Research Committee of the Hungarian Academy of Sciences, and the Jury of Electronics and Electrical Sciences of the Hungarian Research Fund (OTKA). He regularly leads various research projects supported by diverse funds and resources. Many times he served as invited professor in various universities in Italy, Spain, Belgium, and France. 